\author{
ANNALS OF “DUNAREA DE JOS” UNIVERSITY OF GALATI \\ MATHEMATICS, PHYSICS, THEORETICAL MECHANICS \\ FASCICLE II, YEAR X (XLI) 2018, No. 2
}

Article DOI: https://doi.org/10.35219/ann-ugal-math-phys-mec.2018.2.15

\title{
PERCEPTION OF ROMANIAN CONSUMER ON NUTRITION LABELLING OF FOOD PRODUCTS
}

\author{
Adriana Elena Radu ${ }^{1, *}$, Petru Alexe ${ }^{1}$ \\ 1 "Dunarea de Jos" University of Galati, Faculty of Sciences and Environment, Romania, e-mail: \\ adriana.radu@ugal.ro \\ *Corresponding author: adriana.radu@ugal.ro
}

\begin{abstract}
This paper is aimed to present the perception of consumers on the nutrition labelling of food products on the Romanian market in December 2016. The methodology used was a simple survey which was done on 261 consumers from Galati, Romania, who answered different questions in order to observe their perception on nutrition labelling of food products. The questions are closed questions with multiple options of answers. As a conclusion, Romanian consumers seem to be informed about the nutrition labelling and our consumers want to receive more information about the nutrients and healthy benefits of food products.
\end{abstract}

Keywords: consumer research, labelling, nutrition information, Romania.

\section{INTRODUCTION}

Nowadays, all consumers and food industry are very interested in all information which is on the food labelling and in food advertising from different points of view as healthy products, children's products, clean label, marketing, healthy or nutrition claims taking in consideration consumer perception, their culture and their needs.

The purpose of this paper is to identify the perception of consumer on nutrition labelling of food products in Romania market.

Food labelling on the market of Romanian is defined in 2016 by the Romanian Governmental Decision no. 106/2002 [1] and Regulation (EU) No 1169/2011[2] of the European Parliament and of the Council on the provision of food information to consumers. According to Romanian's and EU legislation nutrition and health claim are not mandatory to be on the label of the food products, but if this type of claims should be on the label, they will be compliance with the requirements of Regulation (EC) No 1924/2006 [3] of the European Parliament and of The Council of 20 December 2006 foods. Romanian's food legislation is known as a restricted food legislation at the EU level, when we discuss about pictorial. It is forbidden to use any type of pictorials (real picture, stylized picture or graphic picture), if the products contain only natural or non-natural flavourings. The condition to use a pictorial on the Romanian's food products label is to have the ingredient which is depicted on the label included in the list of ingredients and followed by the percentage in the final product. If we discuss about organic food products this particular labelling is applicable according to the requirements defined in the Council Regulation (EC) No 834/2007 of 28 June 2007 on organic production and the labelling of organic products [4].

Based on Regulation (EU) 1169/2011 [2], the label for the EU market contains such information as: the name of food product, name of food business operator who is responsible for the 
product in EU market, ingredients and processing aids which contain substance causing food allergies or intolerance, the data of durability, list of ingredients, net weight, quantity of the ingredients expressed in percent's for the ingredients which are mentioned in the legal name or are emphasized on the label, special storage conditions, if necessary, instruction of preparation, if required in order to consume proper products, the percent of alcohol for alcoholic beverages with a content above $1.2 \%$ alcohol, the country of origin for products required by specific law, nutrition declaration, information if the product is packaged in a protective atmosphere, etc. Nutrition declaration is mandatory start with December 12, 2016. All food mandatory information on Romania's market is required to be in the local language (i.e. Romania, in our case) and should be mandatory expressed in words and numbers and additional in symbols and pictograms in order to not be misleading for consumers. The additional translation in another language could be added on the pack as optional information.

The label of food products can be seen as a channel to send the information from the producer to the final consumer. $[5,6]$.

Based on the Federal Register [7], the main purpose of the Nutrition Labelling and Education Act of 1990 (NLEA) was to support the consumers' choice and to increase their knowledge "more informed and healthier food choices in the context of their daily diet".

The aim of this paper is to demonstrate that Romanian consumers are more interested in the benefits of the food products and in learning more information about products through the nutrition labelling turned into a friendly tool.

\section{MATERIALS AND METHODS}

A questionnaire was presented directly to 261 male and female consumers from the Romanian town of Galati, in December 2016. The questionnaire had 20 questions with multiple choice answers. The questions were elaborated in a simple way, in order to obtain as accurate answers as possible.

The interrogation was done on the street, near the various supermarket and traditional markets during the first 14 days of December. Around 18 respondents per day have been interrogated. The method used was a simple discussion, in order to collect all information necessary for our study. First, our respondents were asked friendly if they were available to fill in the questionnaire, and only after granting their permission were they provided with the questions and the related answers, in a step by step manner.

The soft used for analysis was IBM SPSS Statistics 20.

\section{RESULTS AND DISCUSSION}

The results of the questionnaires were processed using the SPSS program, which provided descriptive statistics. The demographic characteristic of respondents, such as gender, age and education level are presented in Table 1. From the sum total of the interrogated consumers (259), 5 did not want to answer the question related to their educational level. From 254 respondents of this study, 176 were females and 78 were males. One conclusion is that women are more involved and interested in shopping than men. The highest interest of women in shopping food is also based on the culture of the Romanian consumers, where the woman plays a big role for the meals and education of children. Literature has shown that women are more responsible in the family for the nutritional habits and heath than men. [8].

Studying the information from Table 1, we could see that younger consumers aged between $18 \div 30$ years (approximately $78 \%$ ) are more available to answering the questionnaire, followed by respondents with age ranging $31 \div 50$ years $(20 \%)$ and last, almost $2 \%$ of the consumers are given by respondents with age over 51 years. 
Table 1. Demographic characteristic of respondents.

\begin{tabular}{|c|c|c|c|c|c|c|c|c|}
\hline & & & & \multicolumn{4}{|c|}{ Age } & \multirow[b]{2}{*}{ Total } \\
\hline \multicolumn{4}{|l|}{ Gender } & $18-30$ years & $31-50$ years & $51-65$ years & $>66$ years & \\
\hline \multirow[t]{8}{*}{ Female } & Education & primary school & Count & 0 & 1 & 2 & 1 & 4 \\
\hline & & & $\%$ within Age & $0.0 \%$ & $3.2 \%$ & $50.0 \%$ & $50.0 \%$ & $2.3 \%$ \\
\hline & & high school & Count & 44 & 21 & 0 & 1 & 66 \\
\hline & & & $\%$ within Age & $31.7 \%$ & $67.7 \%$ & $0.0 \%$ & $50.0 \%$ & $37.5 \%$ \\
\hline & & university & Count & 95 & 9 & 2 & 0 & 106 \\
\hline & & & $\%$ within Age & $68.3 \%$ & $29.0 \%$ & $50.0 \%$ & $0.0 \%$ & $60.2 \%$ \\
\hline & Total & & Count & 139 & 31 & 4 & 2 & 176 \\
\hline & & & $\%$ within Age & $100.0 \%$ & $100.0 \%$ & $100.0 \%$ & $100.0 \%$ & $100.0 \%$ \\
\hline \multirow[t]{8}{*}{ male } & Education & primary school & Count & 0 & 2 & & & 2 \\
\hline & & & $\%$ within Age & $0.0 \%$ & $10.0 \%$ & & & $2.6 \%$ \\
\hline & & high school & Count & 18 & 14 & & & 32 \\
\hline & & & $\%$ within Age & $31.0 \%$ & $70.0 \%$ & & & $41.0 \%$ \\
\hline & & university & Count & 40 & 4 & & & 44 \\
\hline & & & $\%$ within Age & $69.0 \%$ & $20.0 \%$ & & & $56.4 \%$ \\
\hline & Total & & Count & 58 & 20 & & & 78 \\
\hline & & & $\%$ within Age & $100.0 \%$ & $100.0 \%$ & & & $100.0 \%$ \\
\hline \multirow[t]{8}{*}{ Total } & Education & primary school & Count & 0 & 3 & 2 & 1 & 6 \\
\hline & & & $\%$ within Age & $0.0 \%$ & $5.9 \%$ & $50.0 \%$ & $50.0 \%$ & $2.4 \%$ \\
\hline & & high school & Count & 62 & 35 & 0 & 1 & 98 \\
\hline & & & $\%$ within Age & $31.5 \%$ & $68.6 \%$ & $0.0 \%$ & $50.0 \%$ & $38.6 \%$ \\
\hline & & university & Count & 135 & 13 & 2 & 0 & 150 \\
\hline & & & $\%$ within Age & $68.5 \%$ & $25.5 \%$ & $50.0 \%$ & $0.0 \%$ & $59.1 \%$ \\
\hline & Total & & Count & 197 & 51 & 4 & 2 & 254 \\
\hline & & & $\%$ within Age & $100.0 \%$ & $100.0 \%$ & $100.0 \%$ & $100.0 \%$ & $100.0 \%$ \\
\hline
\end{tabular}

The level of the consumer's education and understanding of food labelling. Examining the consumer's' level of education we could observe that the respondents with a high level of education are more preoccupied with information which is given through the labelling of food and with the benefits of food products in their diets.

Millions of consumers who want to maintain a healthful diet, in particular those with dietary restriction first look at the information provided by the Nutrition label $[9,10]$.

The trend in obesity has been escalated since 1993, which reflects that without the Nutritional labelling, things could be worse and is not clear which the label is related to obesity rates, because it is not consulted when the consumer purchases food products (Centers for Disease Control and Prevention (CDC 2013); [11].

Legibility of food labelling. In order to determine what is the perception of Romanian's consumers on the visibility of information on the labelling, our respondents were asked if the dimension of the text should have a uniform presentation on the label, how they found the text (i.e. too small, maybe and whether labels with texts difficult to read should receive more details through the additional documents accompanying the products. Figures 1 - 3 present the result of these 
questions and one of conclusion is that the dimension of text should be improved, or maybe the text dimension does not comply with the requirements of the EU legislation.

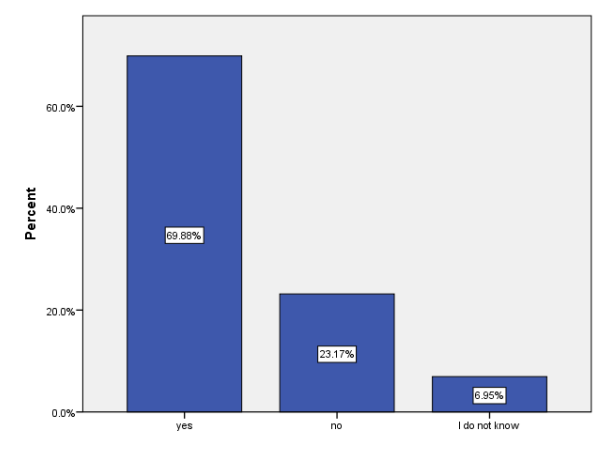
label.

Figure 1. Perception of consumer on information that characters are too small on the

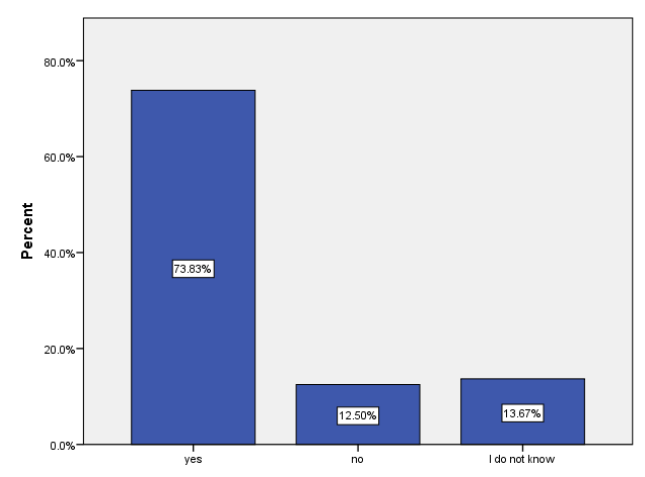

Figure 2. Perception of consumer that characters should be uniformized on the label.

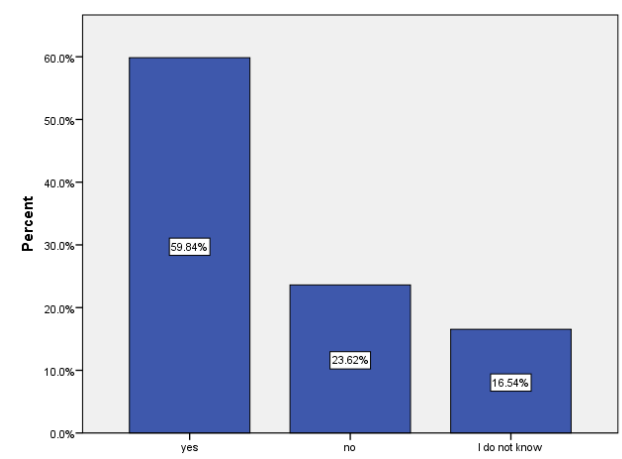

Figure 3. Additional information needed on different material when the characters are too small on the label.

The respondents were also asked to identify the features of the food labelling by choosing one answer from the four available options. Most of the respondents (46\%) declared that they would read the information easily (31\%) confirmed that they found it difficult to read the information, (4\%) declared that the information was very difficult to read and the rest of respondents $(2 \%)$ did not want to answer this question. We could observe a good evolution in that labelling is easy to read in comparison with study done in 28 European countries [12], whose respondents find labelling information very difficult to read (67\%). The reason of this good evolution is the visibility of label which could have the minimum dimension for height of characters that are mandatory and have been implemented through the 
Regulation EU 1169/2011 [2]. This Regulation stipulates that the minimum height of the small letters is $0.9 \mathrm{~mm}$ for the small labels with the surface of minimum or equal with $80 \mathrm{~cm}^{2}$ and $1.2 \mathrm{~mm}$ height for the small characters of the labels with a surface bigger than $80 \mathrm{~cm}^{2}$, which increase the percentage of the food products labelling easy reading.

Different studies also mention that font size of characters is a frequent problem for consumers who check the ingredient list. [12].

Nutrition labelling. The respondents were questioned on how they understood the nutritional labelling on the label, and whether they could read and understand it, by the simple choice of 1 answer from 3 options. The results of our survey showed that $53.3 \%$ of respondents declared that they were partially able to read and understand the nutrition declaration, $34.4 \%$ of the respondents declared that they were able to read and understand it, $10 \%$ of the respondents confirmed that they could not understand the nutrition declaration and $2.3 \%$ of them did not want to answer this question.

Consumers with a higher level of education are more likely to understand and read the nutrition declaration (59\% from $34.3 \%$ of respondents who declared that they understood the nutrition declaration are consumers with a higher level of education). Research shows us that consumers want a front of pack nutrition labelling from which to understand the healthfulness of the product clearly and that this information comes from a trusted source [13].

In order to see what Romanian's habits to choose a food product based on study of the nutrition declaration are, our respondents were asked to identify the score for the nutrition declaration, as a factor of decision in the selection of the food product, on a scale between 1 to 10 . Our results underlined that this preference is not significant; the consumers (in maximum proportion of $13 \div 20 \%$ ) identified the score for the nutrition declaration as a decisional factor to be situated between $5 \div 8$ (Figure 4.). One conclusion could be that in Romania we do not have performing programs for nutrition education.

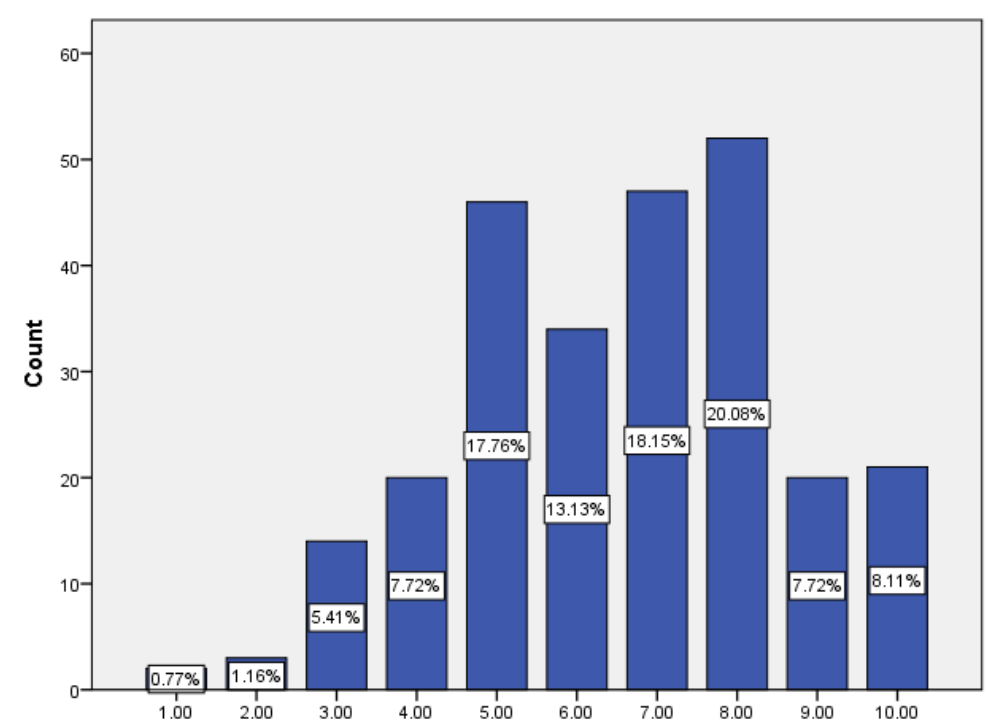

Figure 4. Nutrition declaration as a decision factor in the selection of food products.

We asked respondents if they wanted the nutrition declaration to contain the minimum nutritional information for all food products. The results show us that $80.7 \%$ of the consumers want to have the nutrition declaration for all food products, $15.4 \%$ are indifferent, $3.5 \%$ of the respondents do not want this information on it and $0.4 \%$ did not want to answer this question. One conclusion could be that Romanian consumers are interested in increasing their knowledge about the nutrients in the food products. 
On the question ho, the nutrition declaration should be reported per $100 \mathrm{~g} / 100 \mathrm{ml}$, per unit of consuming or per portion size, our result show the $35.1 \%$ of respondents prefer to have the nutrition declaration per units of consumption , $42.9 \%$ of respondents want to receive this information per portion size, $21.2 \%$ prefer to receive it per $100 \mathrm{~g} / 100 \mathrm{ml}$ and only $0.8 \%$ did not want to answer this questions. According to Regulation EU 1169/2011 [2] the nutrition declaration is mandatory from December 13, 2016 and it should be per $100 \mathrm{~g} / 100 \mathrm{ml}$ and optional per portion size or unit of consumption.

The Nutrition declaration on food products labelling can be considered a cost-effective method for the communication of the nutrition information to consumers, because this data appears at the point of sale for almost all of the packaged food products [14]. Literature mentions that the consumers' perception on the nutrition declaration of food is complex and it does not always have the potential to communicate effectively. [15 - 18].

On the question whether our label should contain information related to daily reference intake of nutrients, $81.1 \%$ of the respondents agreed that such an information should be on the label, $18.5 \%$ did not want to have it and $0.4 \%$ did not want to answer the questions. Based on Regulation EU $1169 / 2011$ [2], if the value for the daily reference intake is given through the labelling, in this case it is mandatory to add the statement "Reference intake of an average adult (8 $400 \mathrm{~kJ} / 2000 \mathrm{kcal})$ "in the same visual field with the nutrition declaration.

Consumers were asked if they wanted to have the nutrition declaration available also for the non-prepacked food. The results show that $58.3 \%$ of our respondents want not to have this information for all products, $33.6 \%$ of the consumers also want to have the nutrition declaration inculded and the difference of $8.1 \%$ do not need it.

The perception of Romanian consumers regarding the front of pack nutrition declaration. The front of pack nutrition declaration is a voluntary system used in different countries from the European and Non-European Union countries.

We asked respondents if they wanted to have the label nutrition declaration under traffic light system, which is used in the UK, where the content of nutrients in big percentages is marked with red, the medium content of nutrients is marked with yellow and lower content of nutrient in the final product is marked with the green color (see Figure 5.). The result underlined that $56 \%$ of the respondents did not want this system on the label, followed by difference who wanted.

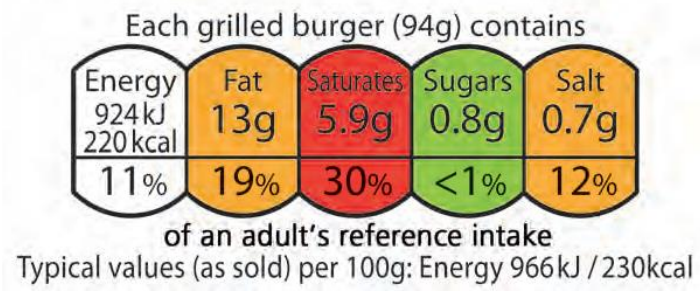

\section{Figure 5. Example of traffic light labelling used in UK. [19]}

A system used as a front of pack nutrition declaration is an icon of nutrient with the value of nutrients related to the reference intake of an average adult. The respondents were also questioned on how they would find nutrition labelling if the content of each nutrient would be reported as a percent from the daily reference intake and the information would be presented under a column format filled with the correct percentage of the nutrient content. Under this format, the nutrition information was found by $81.1 \%$ of our respondent as a representation with clear information, $18.5 \%$ of the respondents found it difficult to read and $0.4 \%$ of the respondents did not want to give any answer.

On the question about the label including the two both versions of nutritional information representation both as a traffic light (UK system) [19] and as a column system / bar system, only $39.4 \%$ of the respondents wanted this information in a double format, $17.8 \%$ did not wanted both versions, $41.3 \%$ declared they did not know and only $1.5 \%$ from respondents did not want to answer to this question. 
On the question related to the presence of the nutrition declaration on the label, on the front of pack or on the back of pack, the result shows that $43.4 \%$ of the respondents want to receive this information on back of pack of label, 35.5\% of the respondents declared that they did not know and $20.8 \%$ of the consumers declared that they wanted it on the front of pack. A survey from the US Food and Drug Administration (FDA) found that $67 \%$ of the respondents reported that they would use front of pack symbols on the nutrition labelling often or sometimes when they were making purchasing decisions, but a non-standardized labelling system would put in the consumers difficulty if they were to make a good evaluation and to compare the nutritional parameters of foods. [20].

Other information on food labelling. The consumers were also asked if they wanted to find on the label information about: the toxic effect of some components, instruction of utilization and recommendations for some healthy products. The results show that $59.5 \%$ of the consumers want to receive all this information, $21.6 \%$ of the respondents want to receive information about toxicity and healthy recommendations and the rest of the interviewed wanted to receive information only for each category in almost the same percent. Based on the EU legislation, only for some ingredients is it mandatory to add different statements on the labelling. For example, for food colours from Annex V from Reg EU 1333/2008 [21], it is mandatory to add the statement "name or E number of the colour(s) - may have an adverse effect on activity and attention in children"; another example is from Annex III of Regulation EU 1169/2011 [2] which stipulates that it is mandatory to add the following statement "High caffeine content. Not recommended for children or pregnant or breast-feeding women' in the same field of vision as the name of the beverage, followed by a reference in brackets for the caffeine content expressed in mg per 100ml" for Beverages with high caffeine content or foods with added caffeine.

Literature (1) mentions that consumers frequently read the ingredient list which is part of the food label. For example, it is reported that $52 \%$ of the interrogated consumers consult the ingredient list, the nutritional label and the claims [10]; a higher percentage (78\%) is mentioned in another study [22].

Literature emphasizes that consumers are vulnerable to food manufacturers, who a highlight healthy aspects of an overall unhealthy product, because people tend to use heuristic-based decision making when they are pressed by time [23].

\section{CONCLUSIONS}

In line with the notion that knowledge-is-power, the findings of this study suggest that nutrition knowledge supports the food nutrition label use. According to specialist literature, nutrition knowledge seems to help the consumer by directing his/her attention to the information which is promoted through labelling or advertising, allowing more accurate information when the decision to choose the product will be done.

Although the review highlights that Romania's consumers are interested in the nutritional benefits of food products and that a tool for improving the nutrition communication through food labels is also needed.

Also, the culture of Romanian consumers should be developed through different communication channels highlighting the benefits of food products.

\section{References}

1. Governmental Decision no. 106/2002 on food labelling amended by the following acts: HG 1822/2005; HG 173/2006; HG 1357/2006; HG 1529/2007; HG 751/2008; HG 685/2009; HG 796/2011;

2. Regulation (EU) no 1169/2011 of the European Parliament and of the council of 25 October 2011 on the provision of food information to consumers, amending Regulations (EC) No 1924/2006 and (EC) No 1925/2006 of the European Parliament and of the Council, and repealing Commission Directive 87/250/EEC, Council Directive 90/496/EEC, Commission 
Directive 1999/10/EC, Directive 2000/13/EC of the European Parliament and of the Council, Commission Directives 2002/67/EC and 2008/5/EC and Commission Regulation (EC) No 608/2004 amended by Commission Delegated Regulation (EU) No 1155/2013 and Commission Delegated Regulation (EU) No 78/2014.

3. Regulation (EC) No 1924/2006 Of The European Parliament and of the Council of 20 December 2006 on nutrition and health claims made on foods

4. Council Regulation (EC) No 834/2007 of 28 June 2007 on organic production and labelling of organic products and repealing Regulation (EEC) No 2092/91 amended by Council Regulation (EC) No 967/2008 of 29 September 2008 and Council Regulation (EU) No 517/2013 of 13 May 2013.

5. Bernues, A., Olaizola, A., \& Corcoran, K. (2003). Labelling information demanded by European consumers and relationships with purchasing motives, quality and safety of meat. Meat Science, 65, 1095e1106.

6. Grunert, K. G., Wills, J. M., \& Fernandez-Celemin, L. (2010). Nutrition knowledge, and use and understanding of nutrition information on food labels among consumers in the UK. Appetite, 55, 177e189.

7. Federal Register (2010), "Front-of-Pack and Shelf Tag Nutrition Symbols; Establishment of Docket: Request for Comments and Information," 75 (82) (April 29), U.S. Department of Health \& Human Services, Food \& Drug Administration, 22602-22606.

8. Zugravu et al. / AUDJG - Food technology (2017), 41(1), 50-61. Attitudes towards food additives: a pilot study Corina Aurelia Zugravu, Elena Narcisa Pogurschi, Daniela Patrascu, Petronela-Diana Iacob1, Carmen G. Nicolae.

9. Lewis, John E., Kristopher L. Arheart, William G. LeBlanc, Lora E. Fleming, David J. Lee, Evelyn P. Davila, et al. (2009), "Food Label Use and Awareness of Nutritional Information and Recommendations Among Persons with Chronic Disease," American Journal of Clinical Nutrition, 90 (5), 1351-57.

10. Ollberding, N. J., Wolf, R. L., \& Contento, I. (2010). Food label use and its relation to dietary intake among US adults. Journal of the American Dietetic Association, 110(8), 1233-1237. doi:10.1016/j.jada.2010.05.007.

11. Choinière, Conrad J. and Amy Lando (2013), “2008 Health and Diet Survey,” U.S. Food and Drug Administration, (accessed March 11, 2014), [available at

http://www.fda.gov/Food/FoodScience

Research/ConsumerBehaviorResearch/ucm193895.htm].

12. Mackey, M. A. and Metz, M. (2009), Ease of reading of mandatory information on Canadian food product labels. International Journal of Consumer Studies, 33: 369-381. doi:10.1111/j.1470-6431.2009.00787.

13. Hawley, Kristy L., Christina A. Roberto, Marie A. Bragg, Peggy J. Liu, Marlene B. Schwartz, and Kelly D. Brownell (2012), "The Science on Front-of-Package Food Labels," Public Health Nutrition, 16 (3), 430-39, [doi:10.1017/S1368980012000754].

14. Campos, S., Doxey, J., \& Hammond, D. (2011). Nutrition labels on pre-packaged foods. A systematic review. Public Health Nutrition, 14(08), 1496-1506. doi:10.1017/S1368980010003290.

15. Drichoutis, A. C., Nayga, J. R. M., \& Lazaridis, P. (2009). Can nutritional label use influence body weight outcomes? Kyklos, 62(4), 500-525. doi:10.1111/j.1467- 6435.2009.00448.x.

16. Hieke, S., \& Taylor, C. R. (2012). A critical review of the literature on nutritional labeling. Journal of Consumer Affairs, 46(1), 120-156. doi:10.1111/j.1745- 6606.2011.01219.x.

17. Lin, C.-T. J., \& Yen, S. T. (2010). Knowledge of dietary fats among US consumers. Journal of the American Dietetic Association, 110(4), 613-618. doi:10.1016/j.jada.2009.12.020.

18. Wills, J., Schmidt, D., Pillo-Blocka, F., \& Cairns, G. (2009). Exploring global consumer attitudes toward nutrition information on food labels. Nutrition Reviews, 67(s1), S102-S106.

19. Guide to creating a front of pack $(\mathrm{FoP})$ nutrition label for pre-packed products sold through retail outlets, 2003, UK. 
20. Choinie`re CJ, Lando AM (2010) 2008 Health and Diet Survey. http://www.fda.gov/Food/ScienceResearch/Research/Areas/ConsumerResearch/ucm193895.ht m (accessed April 2010).

21. Regulation (EC) no 1333/2008 of the European Parliament and of the council of 16 December 2008 on food additives

22. Norazmir, M. N., Norazlanshah, H., Naqieyah, N., \& Anuar, M. I. K. (2012). Understanding and use of food package nutrition label among educated young adults. Pakistan Journal of Nutrition, 11(10), 934-940. doi:10.3923/ pjn.2012.934.940.

23. Schofield H \& Mullainathan S (2008) The psychology of nutrition messages. Adv Health Econ Health Serv Res 19,145-172. 